\title{
p63 Silencing induces reprogramming of cardiac fibroblasts into cardiomyocyte-like cells
}

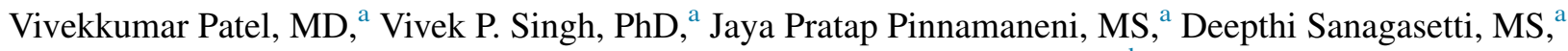
Jacqueline Olive, BS, ${ }^{a}$ Megumi Mathison, $\mathrm{MD}, \mathrm{PhD},{ }^{\mathrm{a}}$ Austin Cooney, $\mathrm{PhD},{ }^{\mathrm{b}}$ Elsa R. Flores, $\mathrm{PhD}$, Ronald G. Crystal, MD, ${ }^{\mathrm{d}}$ Jianchang Yang, MD, PhD, ${ }^{\mathrm{a}}$ and Todd K. Rosengart, $\mathrm{MD}^{\mathrm{a}}$

\section{ABSTRACT}

Objective: Reprogramming of fibroblasts into induced cardiomyocytes represents a potential new therapy for heart failure. We hypothesized that inactivation of p63, a p53 gene family member, may help overcome human cell resistance to reprogramming.

Methods: $p 63$ Knockout $\left(^{-1-}\right)$ and knockdown murine embryonic fibroblasts (MEFs), $p 63^{-1-}$ adult murine cardiac fibroblasts, and human cardiac fibroblasts were assessed for cardiomyocyte-specific feature changes, with or without treatment by the cardiac transcription factors Hand2-Myocardin (HM).

Results: Flow cytometry revealed that a significantly greater number of $p 63^{-/-}$ MEFs expressed the cardiac-specific marker cardiac troponin $\mathrm{T}$ (cTnT) in culture compared with wild-type (WT) cells $(38 \% \pm 11 \%$ vs $0.9 \% \pm 0.9 \%, P<.05)$. HM treatment of $p 63^{-/-}$MEFs increased cTnT expression to $74 \% \pm 3 \%$ of cells but did not induce cTnT expression in wild-type murine embryonic fibroblasts. shRNA-mediated p63 knockdown likewise yielded a 20-fold increase in cTnT microRNA expression compared with untreated MEFs. Adult murine cardiac fibroblasts demonstrated a 200-fold increase in cTnT gene expression after inducible p63 knockout and expressed sarcomeric $\alpha$-actinin as well as cTnT. These $p 63^{-/-}$ adult cardiac fibroblasts exhibited calcium transients and electrically stimulated contractions when co-cultured with neonatal rat cardiomyocytes and treated with HM. Increased expression of cTnT and other marker genes was also observed in p63 knockdown human cardiac fibroblasts procured from patients undergoing procedures for heart failure.

Conclusions: Downregulation of $p 63$ facilitates direct cardiac cellular reprogramming and may help overcome the resistance of human cells to reprogramming. (J Thorac Cardiovasc Surg 2018;156:556-65)

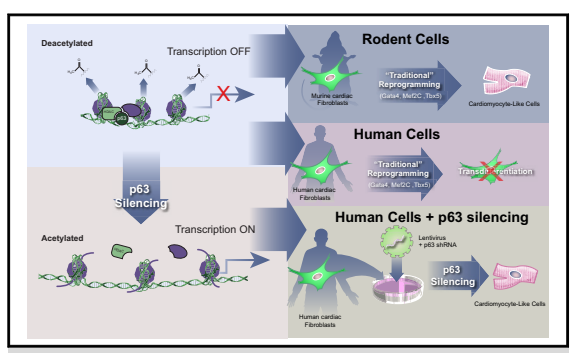

Silencing p63 in cardiac fibroblasts transdifferentiates cardiomyocyte-like cells.

\section{Central Message}

Downregulation of $p 63$ facilitates cardiac cellular transdifferentiation and may help overcome the resistance of human cells to reprogramming.

\section{Perspective}

Recent success reprogramming rodent cardiac cells has been tempered by the relative human cell resistance to reprogramming with currently defined transdifferentiation factors, perhaps due to more rigorous epigenetic constraints in higher-order species. Downregulation of antiplasticity genes such as p63 may enhance cellular plasticity and thereby improve reprogramming efficiency in human cells.

See Editorial Commentary page 566.
Nearly 5 million Americans can be expected to develop advanced congestive heart failure, typically characterized

\footnotetext{
From the ${ }^{a}$ Michael E. DeBakey Department of Surgery, Baylor College of Medicine, Houston, Tex; ${ }^{b}$ Department of Pediatrics, The University of Texas at Austin, Dell Medical School, Austin, Tex; ${ }^{\mathrm{c} D e p a r t m e n t ~ o f ~ M o l e c u l a r ~ O n c o l o g y, ~ M o f f i t t ~ C a n c e r ~}$ Center, Tampa, Fla; and ${ }^{\mathrm{d}}$ Department of Genetic Medicine, Weill Cornell Medical College, New York, NY.

This study was funded by the National Heart Lung and Blood Institute (1R01HL121294-01A1 to T.K.R.).

Drs Yang and Rosengart contributed equally to this article.

Received for publication Dec 6, 2017; revisions received March 6, 2018; accepted for publication March 7, 2018; available ahead of print April 30, 2018.

Address for reprints: Todd K. Rosengart, MD, 1 Baylor Plaza, MS 390, Houston, TX 77030 (E-mail: todd.rosengart@bcm.edu).

$0022-5223 / \$ 36.00$

Copyright (c) 2018 by The American Association for Thoracic Surgery

https://doi.org/10.1016/j.jtcvs.2018.03.162
}

by the replacement of normal contractile cardiomyocytes with fibroblasts and scar tissue after 1 or more episodes of myocardial infarction. ${ }^{1,2}$ Highly invasive surgical procedures such as heart transplant or mechanical circulatory support implantation represent the limited treatment options available for patients with end-stage heart failure, which has traditionally been considered to be an otherwise highly lethal and irreversible condition.

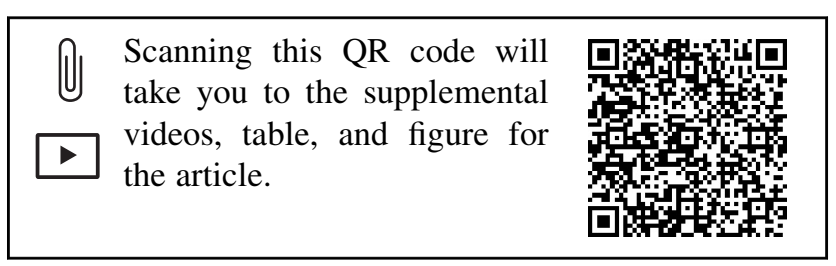




$$
\begin{array}{ll}
\text { Abbreviations and Acronyms } \\
\text { Ad } & =\text { adenovirus } \\
\text { Ad-Cre } & =\text { Ad encoding Cre recombinase } \\
\text { Ad-GFP } & =\text { Ad encoding GFP control vector } \\
\text { BCM } & =\text { Baylor College of Medicine } \\
\text { cTnT } & =\text { cardiac troponin T } \\
\text { DMEM } & =\text { Dulbecco's Modified Eagle Medium } \\
\text { FBS } & =\text { fetal bovine serum } \\
\text { GFP } & =\text { green fluorescent protein } \\
\text { HM } & =\text { Hand2-Myocardin } \\
\text { iCM } & =\text { induced cardiomyocyte } \\
\text { IF } & =\text { immunofluorescence } \\
\text { iPS } & =\text { induced pluripotent stem } \\
\text { MEFs } & =\text { murine embryonic fibroblasts } \\
\text { mRNA } & =\text { microRNA } \\
\text { qRT-PCR } & =\text { quantitative reverse transcriptase } \\
& \text { polymerase chain reaction } \\
\text { shRA } & =\text { short hairpin RNA } \\
\text { WT } & =\text { wild type }
\end{array}
$$

cardiomyocytes by acting as "antiplasticity genes." Given the prohibitive oncogenic risk associated with downregulation of $p 53$, we elected to narrow our focus on strategies for enhancing human cardiac cellular transdifferentiation to the downregulation of $p 63$, a member of the $p 53$ gene family with a reduced oncogenic profile compared with $p 53 .^{22-24}$

\section{METHODS \\ Cell Culture}

Previously characterized p53 knockout $\left(^{-/-}\right)$murine embryonic fibroblasts (MEFs) were gifted from Dr Michelle Barton, MD Anderson Cancer Center. ${ }^{25}$ p63 Knockout $\left(^{-1-}\right)$ MEFs were constructed with a deletion of the DNA binding domain for the $\Delta N-p 63$ and TA-p63 isoforms at exons 6 to 8 of the p63 gene. ${ }^{21,26}$ A stable p63 knockdown MEF cell line was established after p63 lentiviral short hairpin RNA (shRNA) transduction (GE Healthcare Dharmacon, Lafayette, Colo; Clone ID: V3LMM_508694), followed by puromycin selection $(1 \mu \mathrm{g} / \mathrm{mL})$ for 5 to 7 days. All MEFs were cultured in Dulbecco's Modified Eagle Medium (DMEM) supplemented with $15 \%$ fetal bovine serum (FBS), $1 \%$ nonessential amino acids (Cat. No. 11140050; Gibco, Gaithersburg, Md), and $1 \%$ penicillin/streptomycin.

Primary adult mouse cardiac fibroblasts were generated from inducible knockout mice ( $p 63^{f / f l}$, strain C57BL/6, age 8-10 weeks), which have Creactivated loxP sequences flanking the $\Delta N p 63$ gene (Figure E1). ${ }^{21,27}$ These fibroblasts were isolated via a gentleMACS Dissociator (Miltenyi Biotec, Gladbach, Germany) under a protocol approved by the Baylor College of Medicine (BCM) Institutional Animal Care and Use Committee, following which they were cultured on $0.1 \%$ gelatin-coated flasks in Iscove's Modified Dulbecco's Medium (Thermo Fisher Scientific, Waltham, Mass) culture medium supplemented with $20 \%$ FBS, $1 \%$ penicillin/ streptomycin.

Adult human cardiac fibroblasts were harvested from patients undergoing heart transplant or mechanical circulatory support procedures for heart failure (BCM Institutional Review Board approval H-33421; approval date September 25, 2017). The same p63 shRNA lentivirus described previously was used to induce p63 knockdown in these cells. These cells were maintained in FGM Fibroblast Growth Media (CC-4526; Lonza, Walkersville, $\mathrm{Md})$ after infection as previously described. ${ }^{12}$

\section{Vectors}

Lentiviral vectors were prepared from a doublet plasmid encoding Hand2-Myocardin (HM; System Biosciences, Palo Alto, Calif). ${ }^{28}$ Adenovirus (Ad) control vectors encoding green fluorescent protein (GFP), or $\mathrm{Ad}$ vectors encoding Cre and GFP, were obtained from the BCM Vector Development Lab. Viral transduction was performed respectively with multiplicity of infection of 20 (for lentiviruses) or 200 (for Ad) using transfection reagent polybrene ( $8 \mu \mathrm{g} / \mathrm{mL}$; Millipore, Burlington, Mass), based on our experience that these dosages provide the greatest level of transgene expression. After 24 to 48 hours of transfection, media were replaced with reprogramming media consisting of DMEM and M199 supplemented with $10 \%$ FBS, $1 \%$ nonessential amino acids, and $1 \%$ penicillin/streptomycin. All cells were maintained for up to 3 weeks before analysis by flow cytometry, quantitative reverse transcriptase polymerase chain reac-

Flow cytometry. Identification of cardiomyocyte markers via flow cytometry was performed after trypsin detachment of cells from culture plates, after which cells were fixed in $4 \%$ paraformaldehyde and permeabilized in $0.02 \%$ Tween-20, $0.5 \%$ dimethyl sulfoxide in phosphatetion (qRT-PCR), and immunofluorescence (IF).

\section{Cell Characterization Assays}

suppressor genes such as $p 53, p 63, p 21, p 19$, and $p 16$ inhibit iPS cell reprogramming, ${ }^{17-21}$ we speculated that such genes may also impede the transdifferentiation of fibroblasts into reprogramming factors represents a formidable barrier human cellular reprogramming, especially in potential cinical therapeutic applications. ${ }^{14-16}$

In the context of the intriguing observation that tumor

The Journal of Thoracic and Cardiovascular Surgery • Volume 156, Number 2 

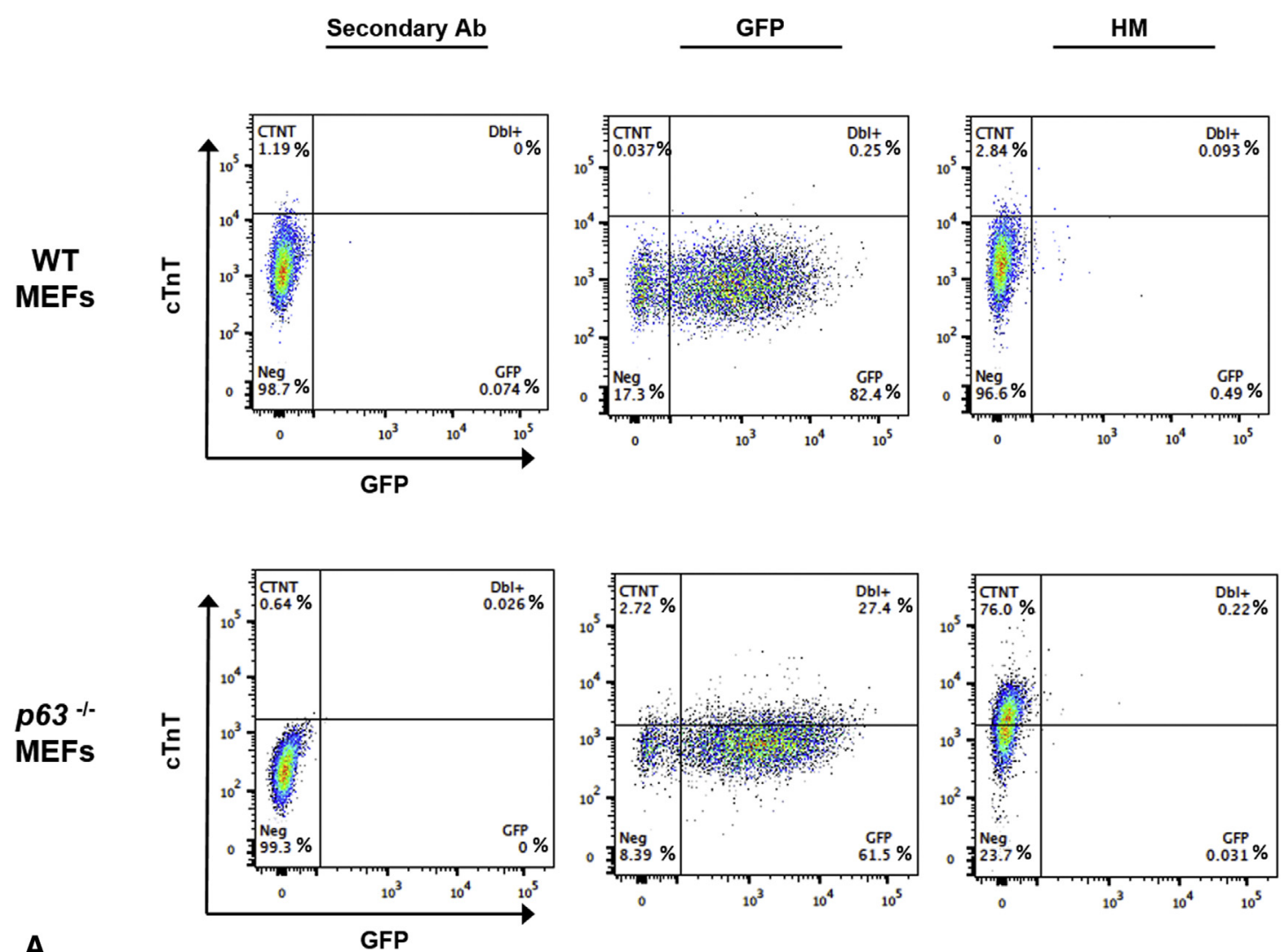

A
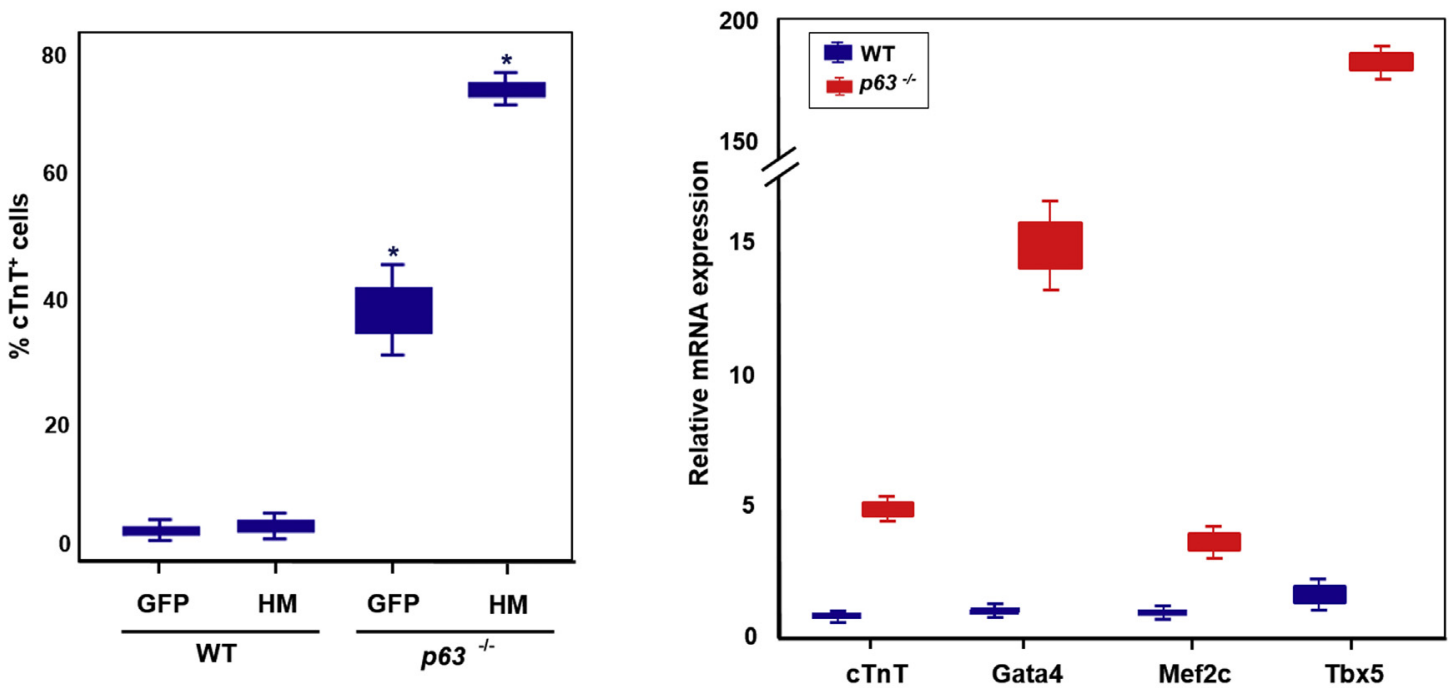

B

C

FIGURE 1. Cardiomyocyte marker expression in MEFs induced by knockout of $p 63$. WT and $p 63^{-/-}$MEFs were treated in vitro with lentiviruses expressing GFP or HM and analyzed 3 weeks later $(\mathrm{n}=2)$. A, Representative flow cytometry plots demonstrating that cTNT expression is increased in $p 63^{-/-}$and HM-treated $p 63^{-/-}$MEFs. B, Percentage of (WT) and $p 63^{-/-}$MEFs cells expressing $\mathrm{cTnT}^{+}$after treatment with lentiviruses expressing GFP or HM. $* P<.05$ versus WT (control). C, qRT-PCR analysis demonstrating increased relative mRNA expression levels of cTnT and the indicated cardiacdifferentiating factors in $p 63^{-1-}$ versus WT MEFs. Gene expression was normalized to glyceraldehyde 3-phosphate dehydrogenase. $* P<.05$ versus WT (control). MEF, Murine embryonic fibroblasts; WT, wild-type; GFP, green fluorescent protein; HM, Hand2-Myocardin; $c T n T$, cardiac troponin T. 
buffered saline. Blocking was then performed with $5 \%$ bovine serum album in permeabilization buffer. The cells were stained with cardiac troponin T (cTnT) antibody (Cat. No. Ab8295; Abcam, Cambridge, United Kingdom) and goat anti-mouse Alexa 647 secondary antibody (Cat. No. Ab150115; Abcam). The cells were analyzed in an LSRFortessa cell sorter (BD Biosciences, Franklin Lakes, NJ) using FlowJo software (FlowJo, LLC, Ashland, Ore).

qRT-PCR. Relevant gene expression was detected via qRT-PCR from total RNA purified from cultured cells using TRIzol reagent (Cat. No, 10296010; Invitrogen, Carlsbad, Calif), following which cDNA was synthesized with Bio-Rad iScript Reverse Transcription Supermix (Cat. No. 1708841; Bio-Rad, Hercules, Calif) on an Eppendorf Mastercycler (Model 6321), following the manufacturer's instruction. qRT-PCR was performed with SYBR Green PCR Master Mix (Cat. No. 4309155; Thermo Fisher Scientific) on a ViiA 7 Real-Time PCR System (Thermo Fisher Scientific). Results were normalized to glyceraldehyde 3-phosphate dehydrogenase. All primer sequences are listed in Table E1.

IF studies. After fixation of cells grown on IF-qualified cover slips (Cat. No. 89015-724; VWR, Radnor, Penn) with 4\% paraformaldehyde, cells were permeabilized with $0.3 \%$ Triton and blocked in $10 \%$ goat serum. IF was performed using antibodies against cTnT (Cat. No. MS295-P0; Thermo Fisher Scientific), $\alpha$-actinin (Cat. No. A7811; Sigma-Aldrich, St Louis, Mo), and cardiac myosin heavy chain (Cat. No. ab15; Abcam). Goat anti-mouse Alexa 568 was used as the secondary antibody (Cat. No. A11004; Thermo Fisher Scientific). Images were captured with a BioTek Cytation 5 Imaging Multi-Mode Reader (BioTek, Winooski, $\mathrm{Vt)}$ at $40 \times$ magnification.

Co-culture and measurements of contractility and calcium transient. Adult murine $p 63^{f / f l}$ cardiac fibroblasts were seeded onto a 6-well plate coated with $0.1 \%$ gelatin at a density of $150,000 / w e l l$. Two days later, the cells were infected with Ad-GFP control or Ad-Cre-GFP, with or without lentivirus encoding HM, and iCM media were applied the next day. ${ }^{12}$ One week later, the cells were harvested and replated at a ratio of 1:4 onto cultures of primary neonatal rat cardiomyocytes. $^{12}$ These had been isolated with the Miltenyi Biotec GentleMACS
Dissociator and seeded at a density of 50,000 cells/well onto $0.1 \%$ gelatin-coated 12-well plates in DMEM, M199, 4\% horse serum, and preconditioned medium obtained from neonatal rat cardiomyocytes. ${ }^{7}$ Cocultured cells were incubated for a total of 7 weeks.

Cell shortening and $\mathrm{Ca}^{2+}$ transients. Myocytes were fieldstimulated at room temperature to contract by using a Grass S5 stimulator through platinum electrodes placed alongside the bath $(1 \mathrm{~Hz}$, bipolar pulses with voltages $50 \%$ above myocyte voltage threshold). ${ }^{29}$ Cells from random fields were videotaped, and motion was digitized on a computer to assess contractility. For $\mathrm{Ca}^{2+}$ signal measurements, cells were loaded with $1.8 \mathrm{mM} \mathrm{Ca}^{2+}$ and $2 \mu \mathrm{mol} / \mathrm{L}$ Fura-2/AM (Life Technologies, Carlsbad, Calif) for 1 hour at room temperature, washed, and incubated for an additional 1 hour to allow de-esterification of the dye, and then alternately excited at 340 and $380 \mathrm{~nm}$ by using a Delta Scan dual-beam spectrophotofluorometer (Photon Technology International Inc, Birmingham, NJ). $\mathrm{Ca}^{2+}$ transients were expressed as the $340 / 380 \mathrm{~nm}$ ratios of the resulting $510-\mathrm{nm}$ emissions. Data were analyzed with Felix software (Photon Technology International Inc).

Cell proliferation and soft agar oncogenicity assays. To assess cell proliferation, $1 \times 10^{4}$ cells were seeded onto a 24 -well plate, and the total number of viable cells was counted daily for 1 week. Adherent cells were detached with trypsin $0.05 \%$, stained with trypan blue, and counted with a Bio-Rad Automated Cell Counter. Soft agar assays were performed as previously described. ${ }^{30}$ Briefly, 10,000 cells were seeded in a 6-well plate containing soft agar, and images were taken 2 weeks after culture $(400 \times)$.

\section{Statistical Analysis}

Data are represented as mean values \pm standard deviation, unless otherwise indicated. Differences between groups were examined for statistical significance by using the Student $t$ test or analysis of variance. We tested the assumption of normality in each group using the Shapiro-Wilk test and Kolmogorov-Smirnov test. We determined that our data were normally distributed in all groups. Then, we applied post-hoc testing after analysis of variance and adjusted our $P$ values with the Bonferroni method. A $P$ value $<.05$ was considered to indicate significance.
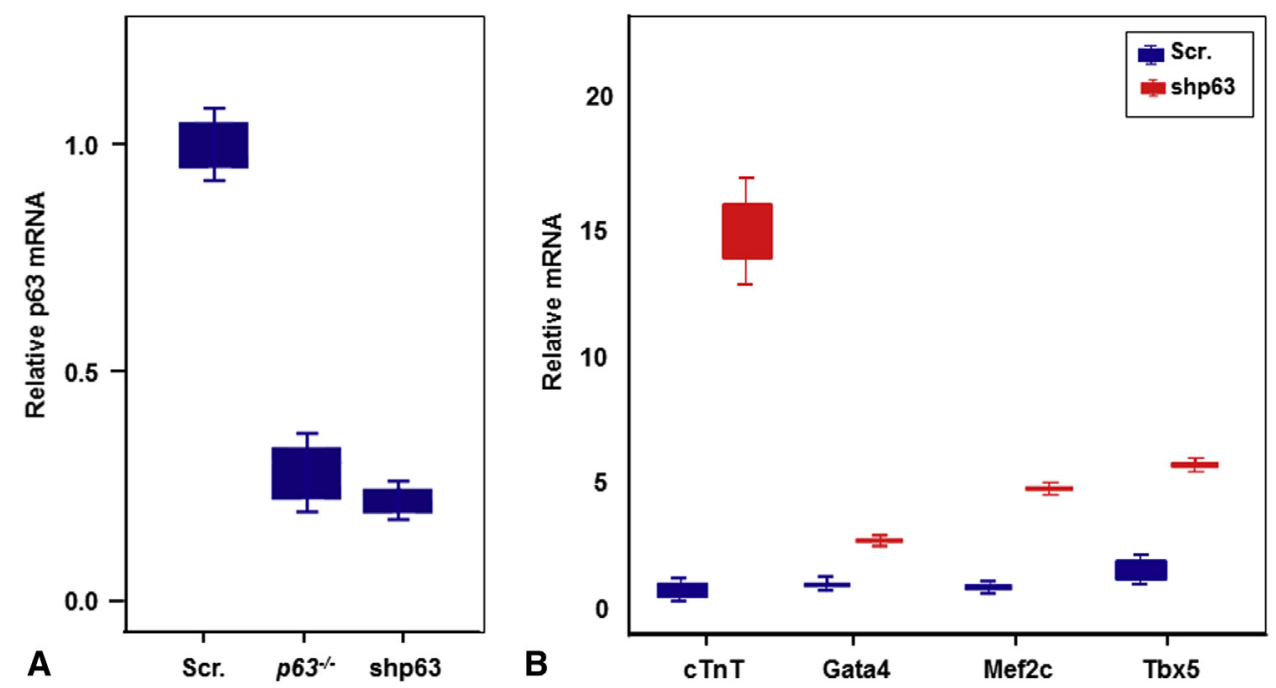

FIGURE 2. Cardiomyocyte marker expression induced by p63 knockdown. A, qRT-PCR analysis demonstrating decreased p63 mRNA expression levels in MEFs after p63 shRNA (shp63) treatment, as described in the Methods, that is equivalent to p63 mRNA levels found in $p 63^{-/-}$MEFs ( $\mathrm{n}=2$ ). B, qRT-PCR analysis demonstrating approximately 20 -fold increase in cTnT expression versus Scr control cells and increased expression of cardiac-transdifferentiating factors in (shp63-treated) p63 knockdown MEFs after 3 weeks of culture. Gene expression was normalized to glyceraldehyde 3-phosphate dehydrogenase. $m R N A$, MicroRNA; $M E F$, murine embryonic fibroblasts; Scr, scrambled shRNA control; $c T n T$, cardiac troponin T. 


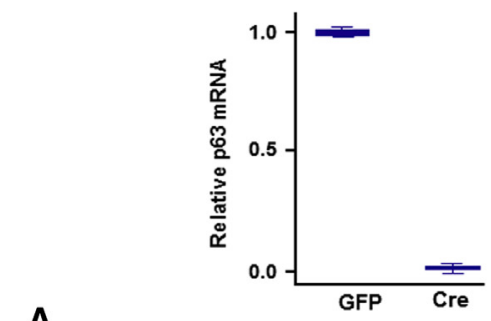

A

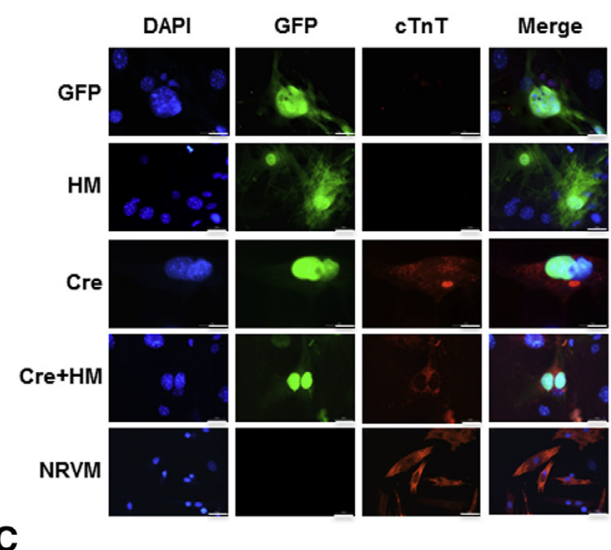

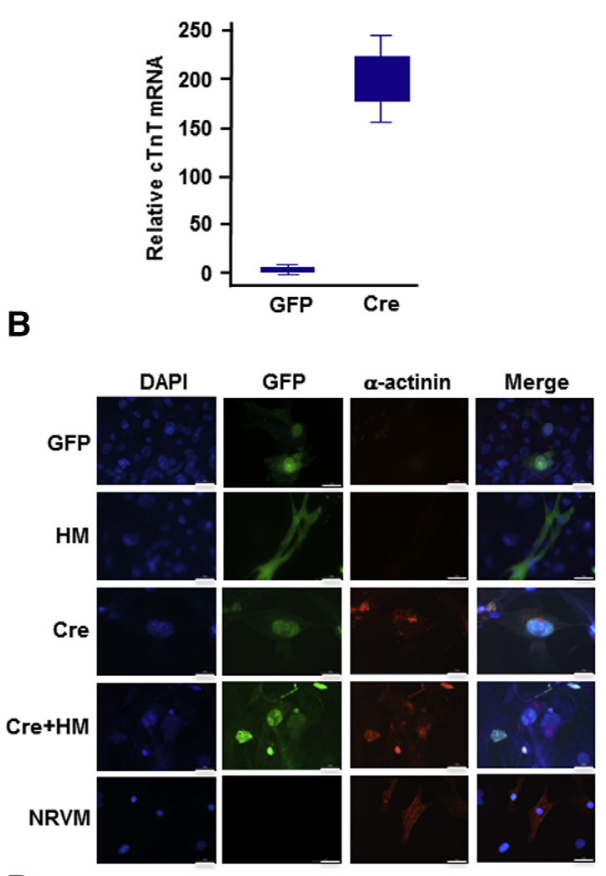

B

D

FIGURE 3. Cardiomyocyte marker expression in adult murine $p 63^{-1-}$ cardiac fibroblasts. A, qRT-PCR analysis showing decreased p63 mRNA expression levels in adult murine cardiac fibroblasts 5 days following Ad-Cre (to induce $p 63$ knockout) versus Ad-GFP administration (vector control) in vitro ( $\mathrm{n}=2$ ). B, qRT-PCR analysis showing an approximately 200-fold increase in cTnT gene expression 3 weeks after Ad-Cre versus Ad-GFP treatment ( $p 63^{-1-}$ vs "WT" cells, respectively [ $=2]$ ). C and D, Representative immunofluorescence images of adult murine cardiac fibroblasts treated as indicated on the left and stained with cardiomyocyte-specific markers cTnT (C) or sarcomeric $\alpha$-actinin (D). Images were captured 3 weeks after Ad-GFP or Ad-Cre treatment, with or without HM cotransduction. The first column depicts the nucleic stain DAPI; second column, GFP fluorescence to identify infected cells; third column, cTnT (C) or $\alpha$-actinin (D); fourth column, merged image. Scale bars represent $30 \mu \mathrm{M}$. NRVM (positive control). Gene expression was normalized to glyceraldehyde 3-phosphate dehydrogenase. Quantitative data are presented as mean $\pm \mathrm{SD}$ from 2 independent repeats. $m R N A$, MicroRNA; Cre, Cre recombinase; GFP, green fluorescent protein; HM, Hand2-Myocardin; DAPI, 4' $6^{\prime}$-diamidino-2-phenylindole; $c T n T$, cardiac troponin T; NRVM, neonatal rat ventricular cardiomyocytes.

\section{RESULTS}

\section{Cardiomyocyte Marker Expression Induced by Downregulation of $p 63$}

To determine whether the $p 53$ gene family could be modulated to enhance cellular reprogramming, we first analyzed cTnT expression in cultures of $p 53^{-1-}$ and wild-type (WT) MEFs. Flow cytometry demonstrated that cTnT was expressed in $12 \% \pm 0.3 \%$ versus $1.2 \% \pm 0.6 \%$ of these cells, respectively $(P<.05)$. We next demonstrated that significantly increased proportions of $p 63^{-/-}$MEFs also expressed cTnT versus WT controls $(38 \% \pm 11 \%$ vs $0.9 \% \pm 0.9 \%$ of cells, $P<.05$; Figure $1, A$ and $B$ ).

When $p 63^{-1-}$ MEFs were treated with HM, the percentage of cells expressing cTnT increased to $74 \% \pm 3 \%$, whereas HM treatment did not induce cTnT expression in WT MEFs. $p 63^{-1-}$ MEFs also demonstrated increased expression of the cardiac-transdifferentiation genes Gata4, Mef2c and Tbx5 (Figure 1, $C$ ), suggesting a potential mechanism of action underlying the cardiodifferentiation of cells with $\mathrm{p} 63$ deletion.
To assess whether (shRNA-mediated) p63 knockdown could also induce $\mathrm{cTnT}^{+}$cardiomyocyte-like cells, we first confirmed that p63 shRNA-treated MEFs demonstrated an appropriate level of reduction of p63 microRNA (mRNA) expression (Figure 2,A). Following this validation, we observed that p63 knockdown in MEFs induced approximately a 20 -fold increase in cTnT mRNA expression and a 3- to 5-fold increase in the mRNA levels of Gata4, Mef2c, and Tbx5 (Figure 2, B), consistent with our observations in $p 63^{-/-}$MEFs.

\section{Adult $p 63^{-/-}$Cardiac Fibroblasts Demonstrated Cardiomyocyte-Like Features}

Having determined that $p 63$ silencing could enhance the transdifferentiation of embryonic cells into cardiomyocytelike cells, we next asked whether p63 downregulation would induce similar changes in adult cells. Adult murine $p 63^{f / f l}$ cardiac fibroblasts were accordingly treated with Ad encoding Cre recombinase (Ad-Cre) or a negative control vector (Ad-GFP). As expected, Ad-Cre administration substantially decreased $p 63$ expression, as verified by 
Ad GFP

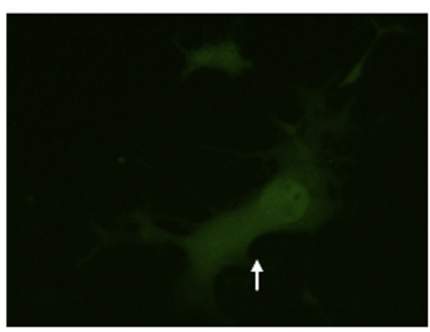

A

Ad GFP

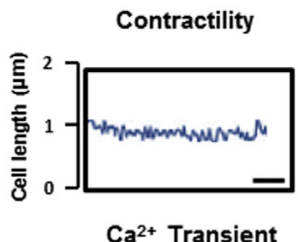

B

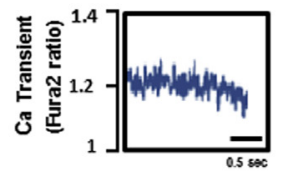

Ad Cre + HM (Cell 1)

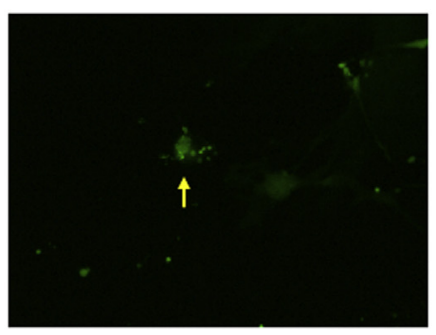

Ad Cre + HM (Cell 1)

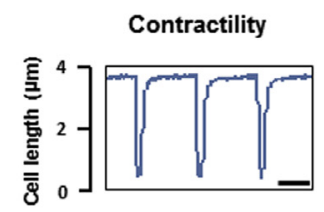

$\mathrm{Ca}^{2+}$ Transient

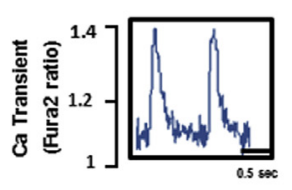

Ad Cre + HM (Cell 2)

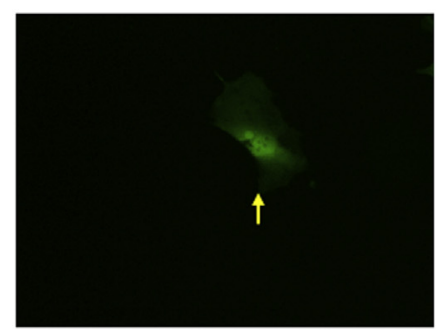

Ad Cre + HM (Cell 2)

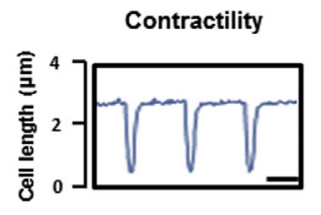

$\mathrm{Ca}^{2+}$ Transient

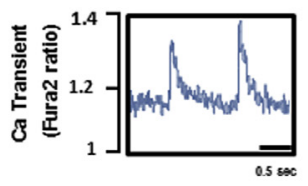

FIGURE 4. Cell contractility and calcium transients induced by $p 63$ knockout and HM treatment in adult murine cardiac fibroblasts, when co-cultured with primary neonatal rat cardiomyocytes. $p 63^{f / f}$ Adult murine cardiac fibroblast treated with Ad-GFP, Ad-Cre, or Ad-Cre and HM (Ad Cre + HM) and were cocultured with neonatal rat cardiomyocytes 1 week after viral transduction for a total of 7 weeks. A, Representative immunofluorescence staining for GFP. White arrow indicates nonbeating $\mathrm{GFP}^{+}$cell; yellow arrow indicates beating $\mathrm{GFP}^{+}$cells, which correspond to Videos 1 and 2. B, Representative contraction and transient peaks corresponding to cells denoted by arrows in same column in A, reflecting cell shortening (contraction) and $\mathrm{Ca}^{2+}$ transients under $1 \mathrm{~Hz}$ frequency and $1.8 \mathrm{mM}$ calcium stimulation. Ad-GFP, Ad encoding GFP control vector; Ad-Cre, Ad encoding Cre recombinase; HM, Hand2-Myocardin.

qRT-PCR (Figure 3, $A$ ). Depletion of $p 63$ in these cells resulted in a 200-fold increase in the expression levels of cTnT (Figure 3, B). IF staining likewise revealed intracellular expression of the cardiac-specific protein markers sarcomeric $\alpha$-actinin and cTnT in these $p 63^{-1-}$ cells but not in cells treated HM alone or in Ad-GFP-treated control cells (Figure 3, $C$ and $D$ ).

To demonstrate that these changes in molecular phenotype corresponded to relevant functional changes in cells with induced $p 63$ silencing, we performed calcium transient and cellular contraction assays on $p 63^{-1-}$ adult murine cardiac fibroblasts co-cultured with neonatal rat cardiomyocytes, as recommended by previous investigators as a means of inducing iCM contractility in vitro. ${ }^{10,12}$ In this analysis, approximately $2 \%$ of the $p 63^{-/}$cardiac fibroblasts treated with HM demonstrated contractions in response to electrical field stimulation, whereas no contractions were observed in cells treated with Ad-GFP or Ad-Cre alone (Figure 4, A, and Videos 1-3). HMtreated $p 63^{-1-}$ cardiac fibroblasts likewise demonstrated calcium transients and cell shortening corresponding to their visual contraction (Figure 4, B).

Several observations support the conclusion that the cells observed to be contracting in these functional studies were the (HM-treated) $p 63^{-/-}$cardiac fibroblasts and rule out the possibility that these findings could represent the artifact of neonatal cardiomyocyte contractions in this preparation. First, cell contractility was not observed when neonatal cardiomyocytes were cultured without the addition of $p 63^{f / f l}$ fibroblasts, consistent with the known loss of this native cardiomyocyte phenotype after the 7 weeks in culture at which time point our contractility studies were undertaken. ${ }^{31}$ Second, contractility was also not seen in wells containing cardiomyocytes co-cultured with Ad-GFPtreated $p 63^{f / f l}$ cardiac fibroblasts (ie, WT control cells), or with (Ad-Cre-treated) $p 63^{-/-}$cells without HM treatment (Figure 4, A, and Videos 1-3). Third, given that contractions were detected only in $\mathrm{GFP}^{+}$(ie, Ad-infected) cells, and that (GFP-labeling) Ad was only administered to $p 63^{f / f l}$ fibroblasts before their introduction into co-culture, it is unlikely that untreated neonatal cardiomyocytes could be mistakenly analyzed as (GFP-labeled) $p 63^{-/-}$cardiac fibroblasts.

\section{Cardiomyocyte Marker Expression in Adult Human Cells With p63 Knockdown}

On the basis of our analysis of the effects of p63 silencing in embryonic and adult rodent fibroblasts, we analyzed the effects of p63 knockdown in (primary culture) adult human 


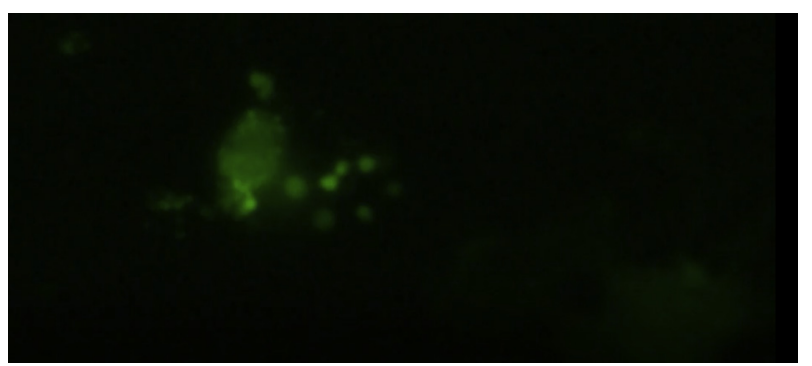

VIDEO 1. $p 63^{-/-}$Adult murine cardiac fibroblasts treated with HM. Contraction was monitored after 7 weeks of co-culture with neonatal rat cardiomyocytes. Contractions are observed in response to external stimulation. Video images correspond to immunofluorescence image of Cell 1 in Figure 4, A. HM, Hand2-Myocardin. Video available at: https://www. jtcvs.org/article/S0022-5223(18)30986-3/fulltext.

cardiac fibroblasts obtained from patients with heart failure. In these studies, p63 knockdown human cardiac fibroblasts demonstrated up to a 30-fold increase in cTnT mRNA expression levels compared with untreated human cardiac fibroblasts (Figure 5).

\section{Minimal Neoplastic Transformation With $p 63$ Knockout}

We also evaluated whether $p 63$ silencing could potentially induce neoplastic transformation, given its homology to $p 53$, using 2 standard assays for this parameter: colony formation in soft agar and in vitro proliferation assays. ${ }^{22-24}$ $p 63^{-/-}$MEFs demonstrated negligibly increased replication rates compared with WT MEFs, whereas $p 53^{-1-}$ MEFs, used as a control comparator, demonstrated significantly increased replication rates (Figure 6, A). Likewise, although $p 53^{-/-}$MEFs grew into

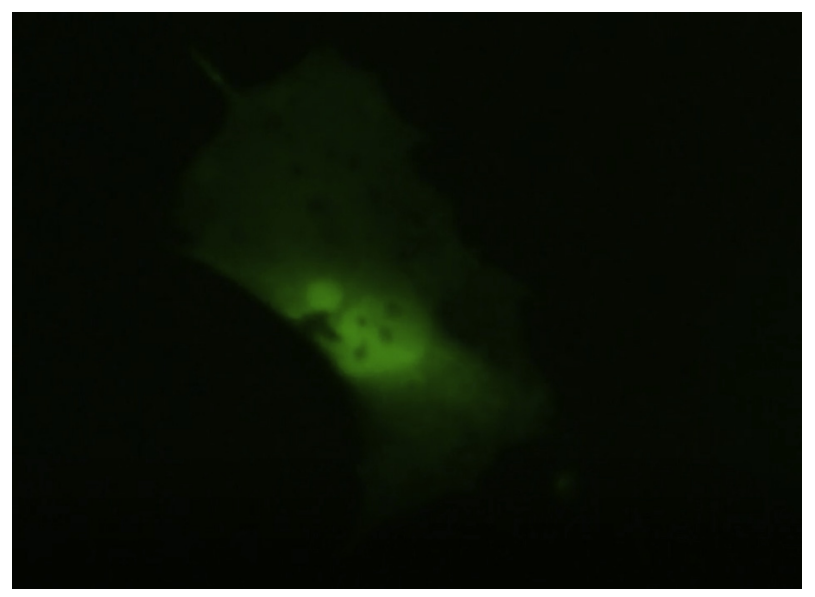

VIDEO 2. $p 63^{-1-}$ Adult murine cardiac fibroblasts treated with HM. Contraction was monitored after 7 weeks of co-culture with neonatal rat cardiomyocytes. Contractions are observed in response to external stimulation. Video images correspond to immunofluorescence image of Cell 2 in Figure 4, A. HM, Hand2-Myocardin. Video available at: https://www. jtcvs.org/article/S0022-5223(18)30986-3/fulltext.

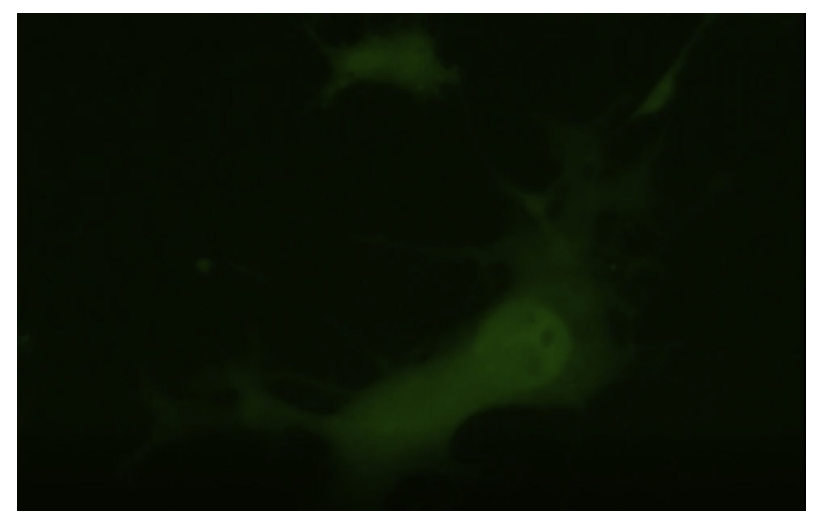

VIDEO 3. $p 63^{f l f l}$ Cells treated with Ad-GFP (wild-type control). Evidence of cell contraction was not detected in response to external stimulation after 7 weeks of co-culture with neonatal rat cardiomyocytes. $H M$, Hand2-Myocardin. Video available at: https://www.jtcvs.org/article/ S0022-5223(18)30986-3/fulltext.

large colonies in soft agar cultures, a marker of oncogenicity, the $p 63^{-/-}$MEFs produced smaller colonies that were comparable with those of WT cells (Figure 6, $B$ ).

\section{DISCUSSION}

Given the now well-documented barriers to the reprogramming of human cells compared with that of lower-order species, we sought to enhance the susceptibility of cells to reprogramming as an alternative to the use of expanded reprogramming cocktail mixtures used by others to overcome this challenge. ${ }^{8,10-16}$ We were able to demonstrate that the transdifferentiation of both rodent and human fibroblasts into cardiomyocyte-like cells was enhanced by the downregulation of $p 63$, a member of the cell senescence family of genes of which $p 53$ is the prototype. This approach was stimulated by the observation that downregulation of either of these genes enhance iPS reprogramming, suggesting they serve as "antiplasticity" genes that may normally suppress excessive or promiscuous gene activation. ${ }^{18-22}$

Given the large number of human neoplasms associated with $p 53$ mutations, we elected to target $p 63$, which has been associated with a far smaller human oncogenic profile than has $p 53 .^{23,24}$ We did not explore the reprogramming effects of other p53 family members such as $p 73$, which have not been described to have antiplasticity effects like $p 53$ and $p 63 .{ }^{24}$ Encouragingly, $p 63$ downregulation appears to be even more effective than $p 53$ downregulation in enhancing cellular reprogramming (Figure 1), consistent with observations that $p 63$ is a potent antiplasticity factor. ${ }^{21}$ Likewise, $p 63$ downregulation resulted in cTnT expression levels 4-fold greater than that which we have observed using optimized reprogramming cocktails on WT cells., ${ }^{9,32}$

Given the apparent potency of $p 63$ silencing, it is prudent to first ask whether $p 63$ downregulation incurs the risk of neoplastic transformation, despite the lack of evidence of 
this risk in the clinical literature. ${ }^{23,24}$ Consistent with previous studies, encouragement can be derived from our observations of a lack of such oncogenicity effects with p63 versus p53 downregulation in soft agar and cell replication assays-the "gold standards" for in vitro neoplastic transformation. ${ }^{23,24,33}$ Additional relevant safeguards may be found in the limited duration of $p 63$ downregulation that is apparently needed to enhance reprogramming (as can be provided with Ad vectors), ${ }^{28}$ and the ability to deliver p63 shRNA locally to cardiac tissues, which naturally have a very low baseline predilection to malignancy. It may also be possible to narrow therapy to a single isoform of $p 63$ or to a downstream effector of $p 63$, such as DCGR8. ${ }^{21}$ Notwithstanding these assurances, more rigorous in vivo validation of these safety data will certainly be needed before clinical applications are considered.

Our findings of the potency of $p 63$ silencing on cardiac fibroblast transdifferentiation is surprising, given that $p 63$ has been previously associated primarily with epithelial development. ${ }^{21,23,24}$ In this context, it is interesting to consider that the spontaneous differentiation of $p 63^{-/-}$ MEFs into cardiomyocyte-like cells observed in our studies may represent a "default" differentiation pattern, consistent with the heart being the first organ formed in embryonic development. ${ }^{34}$ In this paradigm, once $p 63$ gene repression is released, cells revert to a predominant cardiomyocyte gene expression pathway, and the addition of additional cardio-differentiating genes like HM simply enhance this endogenous pathway. ${ }^{7,8,10-12}$
The molecular mechanism underlying cellular cardiodifferentiation mediated by p63-silencing may be related to our observation of the upregulation of the cardiacreprogramming genes Gata4, Mef2c, and Tbx5 in p63deficient cells (Figures 1 and 2); specifically, that p63 repression allows for the increased expression of these otherwise-repressed cardio-differentiating genes. The observation that exogenous Gata4, Mef2c, Tbx 5 together with HM can substitute for $p 63$ downregulation in inducing human cellular reprogramming is consistent with these data. ${ }^{10-12}$ This premise is supported by our findings of effective cardiac reprogramming with $p 63$ downregulation in adult human cardiac fibroblasts as well as embryonic and adult rodent cells. On the other hand, it is possible that p63 downregulation also activates genes, such as the Wnt and B-catenin signaling pathways, which have been shown to be modulated by p63 expression and facilitate cardiac cellular reprogramming. ${ }^{13}$

Although we have not yet identified the specific epigenetic processes by which $p 63$ downregulation leads to enhanced gene activation and cardiodifferentiation, one recently discovered pathway involves the complexing of p63 with histone deacetylases to induce chromatin condensation and the rendering of various gene promoter sites inaccessible to activator complexes. ${ }^{35}$ Conceivably, release of $p 63$ repression may thus allow access to these otherwise excessively repressed promoter sites in human cells.
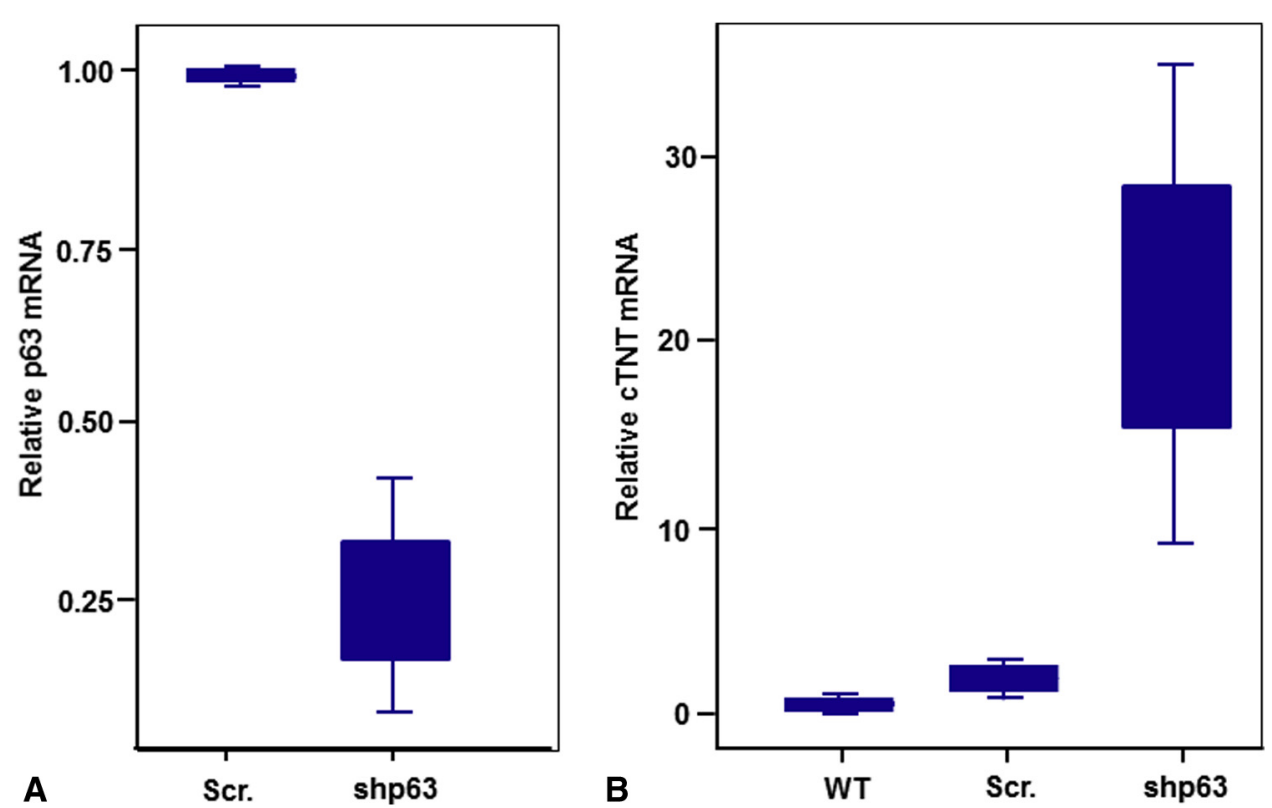

FIGURE 5. Cardiomyocyte marker expression in adult human cells treated with shp63. A, qRT-PCR analysis showing decreased p63 mRNA expression levels in shp63-treated versus scrambled shRNA treated human cardiac fibroblasts, obtained from patients undergoing heart transplant or circulatory support implants, as detailed in the Methods $(\mathrm{n}=2)$. B, Human cardiac fibroblasts procured as described in the Methods demonstrating increased cTnT mRNA expression levels 7 days after treatment with shp63 as compared with cells treated with a scrambled shRNA control (Scr.). Gene expression was normalized to glyceraldehyde 3-phosphate dehydrogenase $(\mathrm{n}=2)$. $m R N A$, MicroRNA; $c T n T$, cardiac troponin $\mathrm{T}$. 


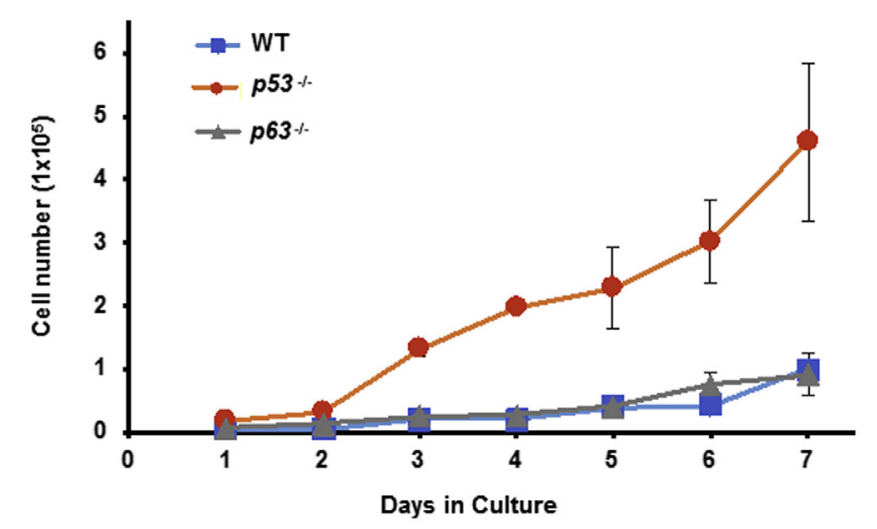

A

WT

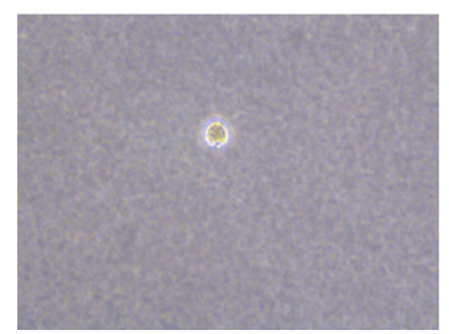

$p 53^{-1-}$

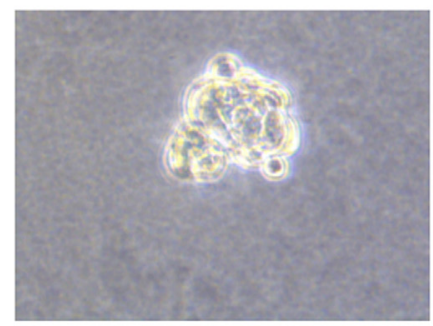

$p 63^{-1-}$

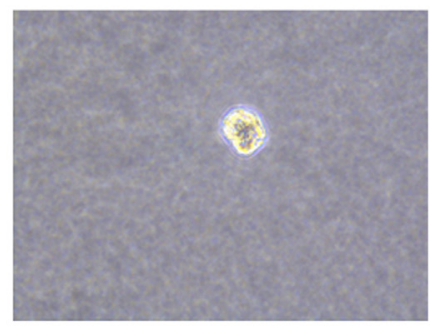

B

FIGURE 6. Neoplastic transformation assessment assays. A, WT, $p 53^{-/-}$, and $p 63^{-/-}$MEFs $\left(1 \times 10^{4}\right.$ cells $)$ were seeded into a 24 -well plate, and the total number of cells was counted daily for a total of 7 days. An increased proliferation rate was observed in $p 53^{-1-}$ but not in $p 63^{-1-}$ MEFs compared with WT cells $(\mathrm{n}=3)$. B, Representative images of soft agar assays, performed as described in the Methods, comparing anchorage-independent growth of WT, $p 53^{-/-}$, and $p 63^{-/-}$MEFs, demonstrating markedly larger $p 53^{-/-}$colonies compared with WT and $p 63^{-/-}$cells. WT, Wild type.

Taken together, the findings of the present study suggest that downregulation of $p 63$ may be a safe and effective strategy for inducing human cardiac cellular reprogramming as a potential therapeutic strategy for the treatment of heart failure. This strategy seeks to overcome barriers to human cellular reprogramming by increasing target cell susceptibility to this process, as compared with present strategies that largely rely on adding a greater number of reprogramming factors to treatment cocktails. The latter may prove to be unduly complex and cumbersome for potential clinical application.

\section{Study Limitations}

Limitations of the current study include our lack of a more expansive analysis of factors other than HM that might optimally enhance cardiac reprogramming, an expanded analysis of additional potential mechanisms of action such as histone deacetylase repression or cellular apoptosis, and our use of a relatively limited, albeit previously validated panel of cardiomyocyte markers (ie, cTnT, $\alpha$-actinin, and myosin heavy chain) to define a cardiomyocyte-like phenotype.

Furthermore, although we did demonstrate critically important physiologic cardiomyocyte markers (ie, calcium transients and cell contraction) in p63downregulated cells, co-culture of these cells with neonatal cardiomyocytes was needed to induce this phenotype. This is consistent with the use of this requisite reprogramming methodology in other in vitro reprogramming studies, ${ }^{12}$ presumably because of the need for an appropriate cardiac "milieu" to induce reprogramming, as suggested by greater efficacy of reprogramming effects observed in in vivo versus in vitro studies. ${ }^{7-12}$ The aforementioned considerations also apply to the relatively low efficiency of reprogrammed cells demonstrating a "full" (contractile) phenotype, which previous studies suggest might be more robust in the in vivo cardiac milieu. ${ }^{7-12}$

Finally, although our use of human cardiac fibroblasts is encouraging in terms of the ultimate clinical applicability of this proposed human cardiac reprogramming strategy, it must be recognized that the cell-culture process inherently selects out the "healthier" cells that can survive the explantation and in vitro culture propagation process. It is therefore not possible to definitively extrapolate these in vitro findings to potential results of in situ cell reprogramming that will ultimately require in vivo testing data that we now plan to perform. 


\section{Conflict of Interest Statement}

Authors have nothing to disclose with regard to commercial support.

We thank Dr Michelle Barton from MD Anderson Cancer Center for the $p 53$ knockout MEF cell line, Payal Raulji from the Flores Lab for her assistance with p63-related cell lines, Jacob Klein for help with oncologic studies, and Qianzi Zhang for her help with statistical analysis. We also thank the BCM Cytometry and Cell Sorting Core and the Integrated Microscopy Core.

\section{References}

1. Shinde AV, Frangogiannis NG. Fibroblasts in myocardial infarction: a role in inflammation and repair. J Mol Cell Cardiol. 2014;70:74-82.

2. Yancy CW, Jessup M, Bozkurt B, Butler J, Casey DE Jr, Drazner MH, et al. 2013 ACCF/AHA guideline for the management of heart failure: executive summary: a report of the American College of Cardiology Foundation/American Heart Association Task Force on practice guidelines. Circulation. 2013; 128:1810-52

3. Kittleson MM, Kobashigawa JA. Management of the ACC/AHA Stage D patient: cardiac transplantation. Cardiol Clin. 2014;32:95-112. viii.

4. Ieda M, Fu JD, Delgado-Olguin P, Vedantham V, Hayashi Y, Bruneau BG, et al. Direct reprogramming of fibroblasts into functional cardiomyocytes by defined factors. Cell. 2010;142:375-86.

5. Qian L, Huang Y, Spencer CI, Foley A, Vedantham V, Liu L, et al. In vivo reprogramming of murine cardiac fibroblasts into induced cardiomyocytes. Nature. 2012;485:593-8.

6. Takahashi K, Tanabe K, Ohnuki M, Narita M, Ichisaka T, Tomoda K, et al. Induction of pluripotent stem cells from adult human fibroblasts by defined factors. Cell. 2007;131:861-72.

7. Song K, Nam YJ, Luo X, Qi X, Tan W, Huang GN, et al. Heart repair by reprogramming non-myocytes with cardiac transcription factors. Nature. 2012;485: 599-604.

8. Nam YJ, Song K, Luo X, Daniel E, Lambeth K, West K, et al. Reprogramming of human fibroblasts toward a cardiac fate. Proc Natl Acad Sci USA. 2013;110: 5588-93.

9. Mathison M, Gersch RP, Nasser A, Lilo S, Korman M, Fourman M, et al. In vivo cardiac cellular reprogramming efficacy is enhanced by angiogenic preconditioning of the infarcted myocardium with vascular endothelial growth factor. $J \mathrm{Am}$ Heart Assoc. 2012;1:e005652.

10. Wada R, Muraoka N, Inagawa K, Yamakawa H, Miyamoto K, Sadahiro T, et al. Induction of human cardiomyocyte-like cells from fibroblasts by defined factors. Proc Natl Acad Sci USA. 2013;110:12667-72

11. Fu JD, Stone NR, Liu L, Spencer CI, Qian L, Hayashi Y, et al. Direct reprogramming of human fibroblasts toward a cardiomyocyte-like state. Stem Cell Rep. 2013;1:235-47.

12. Singh VP, Mathison M, Patel V, Sanagasetti D, Gibson BW, Yang J, et al. MiR590 promotes transdifferentiation of porcine and human fibroblasts toward a cardiomyocyte-like fate by directly repressing specificity protein 1. J Am Heart Assoc. 2016:5:e03922.

13. Ebrahimi B. Reprogramming barriers and enhancers: strategies to enhance the efficiency and kinetics of induced pluripotency. Cell Regen (Lond). 2015;4:10.

14. Vaseghi H, Liu J, Qian L. Molecular barriers to direct cardiac reprogramming. Protein Cell. 2017:8:724-34.

15. Addis RC, Ifkovits JL, Pinto F, Kellam LD, Esteso P, Rentschler S, et al. Optimization of direct fibroblast reprogramming to cardiomyocytes using calcium activity as a functional measure of success. J Mol Cell Cardiol. 2013;60: 97-106.

16. Zhou H, Dickson ME, Kim MS, Bassel-Duby R, Olson EN. Akt1/protein kinase B enhances transcriptional reprogramming of fibroblasts to functional cardiomyocytes. Proc Natl Acad Sci USA. 2015;112:11864-9.
17. Kawamura T, Suzuki J, Wang YV, Menendez S, Morera LB, Raya A, et al. Linking the p53 tumour suppressor pathway to somatic cell reprogramming. Nature. 2009;460:1140-4.

18. Bao X, Wu H, Zhu X, Guo X, Hutchins AP, Luo Z, et al. The p53-induced lincRNA-p21 derails somatic cell reprogramming by sustaining H3K9me3 and CpG methylation at pluripotency gene promoters. Cell Res. 2015;25:80-92.

19. Hong H, Takahashi K, Ichisaka T, Aoi T, Kanagawa O, Nakagawa M, et al. Suppression of induced pluripotent stem cell generation by the p53-p21 pathway. $\mathrm{Na}$ ture. 2009;460:1132-5

20. Itahana K, Zou Y, Itahana Y, Martinez JL, Beausejour C, Jacobs JJ, et al. Control of the replicative life span of human fibroblasts by $\mathrm{p} 16$ and the polycomb protein Bmi-1. Mol Cell Biol. 2003;23:389-401.

21. Chakravarti D, Su X, Cho MS, Bui NH, Coarfa C, Venkatanarayan A, et al. Induced multipotency in adult keratinocytes through downregulation of DeltaNp63 or DGCR8. Proc Natl Acad Sci USA. 2014;111: E572-81.

22. Rasmussen MA, Holst B, Tumer Z, Johnsen MG, Zhou S, Stummann TC, et al. Transient p53 suppression increases reprogramming of human fibro blasts without affecting apoptosis and DNA damage. Stem Cell Rep. 2014 3:404-13.

23. Flores ER. The roles of p63 in cancer. Cell Cycle. 2007:6:300-4.

24. Venkatanarayan A, Raulji P, Norton W, Flores ER. Novel therapeutic interventions for p53-altered tumors through manipulation of its family members, p63 and p73. Cell Cycle. 2016;15:164-71.

25. Kurinna S, Stratton SA, Coban Z, Schumacher JM, Grompe M, Duncan AW, et al. p53 regulates a mitotic transcription program and determines ploidy in norma mouse liver. Hepatology. 2013;57:2004-13.

26. Venkatanarayan A, Raulji P, Norton W, Chakravarti D, Coarfa C, Su X, et al. IAPP-driven metabolic reprogramming induces regression of p53-deficient tumours in vivo. Nature. 2015;517:626-30.

27. Yang A, Schweitzer R, Sun D, Kaghad M, Walker N, Bronson RT, et al. p63 is essential for regenerative proliferation in limb, craniofacial and epithelial development. Nature. 1999;398:714-8.

28. Mathison M, Singh VP, Chiuchiolo MJ, Sanagasetti D, Mao Y, Patel VB, et al. In situ reprogramming to transdifferentiate fibroblasts into cardiomyocytes using adenoviral vectors: Implications for clinical myocardial regeneration. $J$ Thorac Cardiovasc Surg. 2017;153:329-39.e3.

29. Singh VP, Rubinstein J, Arvanitis DA, Ren X, Gao X, Haghighi K, et al. Abnormal calcium cycling and cardiac arrhythmias associated with the human Ser96Ala genetic variant of histidine-rich calcium-binding protein. J Am Heart Assoc. 2013;2:e000460.

30. Nannenga B, Lu X, Dumble M, Van Maanen M, Nguyen TA, Sutton R, et al. Augmented cancer resistance and DNA damage response phenotypes in PPM1D null mice. Mol Carcinog. 2006;45:594-604.

31. Nguyen PD, Hsiao ST, Sivakumaran P, Lim SY, Dilley RJ. Enrichment of neonatal rat cardiomyocytes in primary culture facilitates long-term maintenance of contractility in vitro. Am J Physiol Cell Physiol. 2012;303:C1220-8.

32. Mathison M, Singh VP, Gersch RP, Ramirez MO, Cooney A, Kaminsky SM, et al "Triplet" polycistronic vectors encoding Gata4, Mef2c, and Tbx5 enhances postinfarct ventricular functional improvement compared with singlet vectors J Thorac Cardiovasc Surg. 2014;148:1656-64.e2.

33. Guo X, Keyes WM, Papazoglu C, Zuber J, Li W, Lowe SW, et al. TAp63 induces senescence and suppresses tumorigenesis in vivo. Nat Cell Biol. 2009;11:1451-7.

34. Giacomelli E, Mummery CL, Bellin M. Human heart disease: lessons from human pluripotent stem cell-derived cardiomyocytes. Cell Mol Life Sci. 2017;74 3711-39.

35. Ratovitski EA. Tumor protein p63/microRNA network in epithelial cancer cells Curr Genomics. 2013;14:441-52.

Key Words: cellular reprogramming, transdifferentiation, heart failure, myocardial infarction, regenerative medicine, induced-cardiomyocytes (iCMs) 


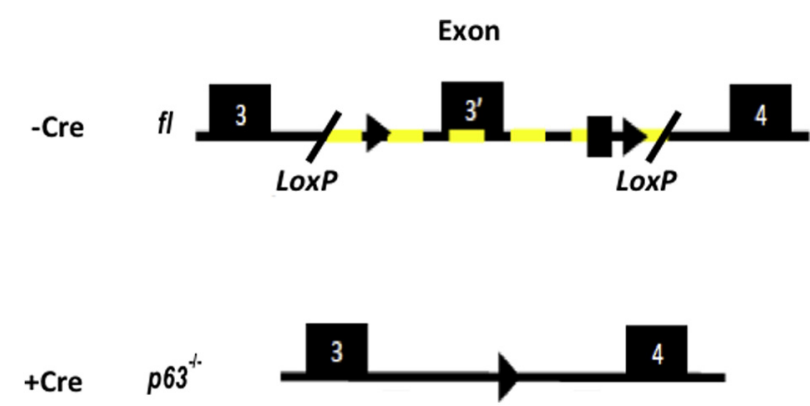

FIGURE E1. Schematic diagram depicting $p 63^{f l / f}$ inducible knockout mouse model. $p 63^{f / f l}$ inducible knockout mice were generated using the Cre-loxP system, as described previously. ${ }^{26}$ LoxP sequences, which recognize Cre, flank the $3^{\prime}$ exon of the $p 63$ gene. The addition of Cre to $p 63^{f / f l}$ removes the targeted region (highlighted in yellow) through recombination, rendering a $63^{-/-}$phenotype.

TABLE E1. qRT-PCR primer sequences for murine and human samples

\begin{tabular}{lll}
\hline & \multicolumn{1}{c}{ Forward primer } & Reverse primer \\
\hline Mouse & & GCTAAGCAGTTGGTGGTGCA \\
GAPDH & TCACCACCATGGAGAAGGC & TGGCAAACAAAAATGGCTTA \\
p63 & CTGGTATTTTTTGTTATGGGCT & CTTCTTCCTGTTCCTCCTCGT \\
cTnT & GTGTGCAGTCCCTGTTCAGA & CATGGCCCCACAATTGAC \\
Gata 4 & GGAAGACACCCCAATCTCG & CGTGGTGTGTGTGGGTATC \\
Mef2c & TCTGCCCTCAGTCAGTTGG & CACCTTCACTTTGTAACTAGGAA \\
Tbx5 & CGAAGTGGGCACAGAGATG & \\
Human & & GGTGGTCCAGGGGTCTTACT \\
GAPDH & CAACGACCACTTTGTCAAGC \\
Dnp63 & GAAAACAATGCCCAGACTCAA & TGCGCGTGGTCTGTGTTA \\
cTnT & AGACGCCTCCAGGATCTGT & TCTTCAACAGCTGCTTCTTCC \\
Gata4 & TTCCAGCAACTCCAGCAA & TCGCACTGACTGAGAACGTC \\
Mef2c & TGATCAGCAGGCAAAGATTG & TGGACACTGGGATGGAGACT \\
Tbx5 & CCAGGAGCATAGCCAAATTTAC & AGGGCTTCTTATAGGGATGGTC \\
Hand2 & GAAGACCGACGTGAAAGAGG & TTCTTGTCGTTGCTGCTCAC \\
Myocardin & AGGGCACAGGGTCTCCTC & GGAAGAATGGGGAGAGAAGC \\
MYH6 & AAGAATGTGCATGAGCTGGA & GATCTTGCCCTCCTCGTG \\
MYH7 & ACACCCTGACTAAGGCCAAA & ATGCGCACCTTCTTCTCTTG \\
Nkx 2.5 & AGTGTGCGTCTGCCTTTC & GTTGTCCGCCTCTGTCTTC \\
Is1 & GCTCTGCTTCTGTACTGCCA & GGGGATGGATTTGCTCTTT \\
\hline
\end{tabular}

GAPDH, Glyceraldehyde 3-phosphate dehydrogenase; $c T n T$, cardiac troponin T. 